\title{
Novel approach to the synthesis of 3-amino-4-arylpyridin-2(1H)-one derivatives
}

\author{
Vladislav Yu. Shuvalov ${ }^{1}$, Sergei A. Chernenko ${ }^{1}$, Anton L. Shatsauskas ${ }^{1}$, \\ Anna L. Samsonenko ${ }^{2}$, Maksim V. Dmitriev ${ }^{3}$, Alexander S. Fisyuk ${ }^{1,2 *}$ \\ ${ }^{1}$ Omsk State Technical University, \\ 11 Mira Ave., Omsk 644050, Russia \\ ${ }^{2}$ Dostoevsky Omsk State University, \\ 55a Mira Ave., Omsk 644077, Russia; e-mail: fisyuk@chemomsu.ru \\ ${ }^{3}$ Perm State University, \\ 15 Bukireva St., Perm 614990, Russia
}

Translated from Khimiya Geterotsiklicheskikh Soedinenii, 2021, 57(7/8), 764-771

Submitted July 15, 2021

Accepted August 5, 2021

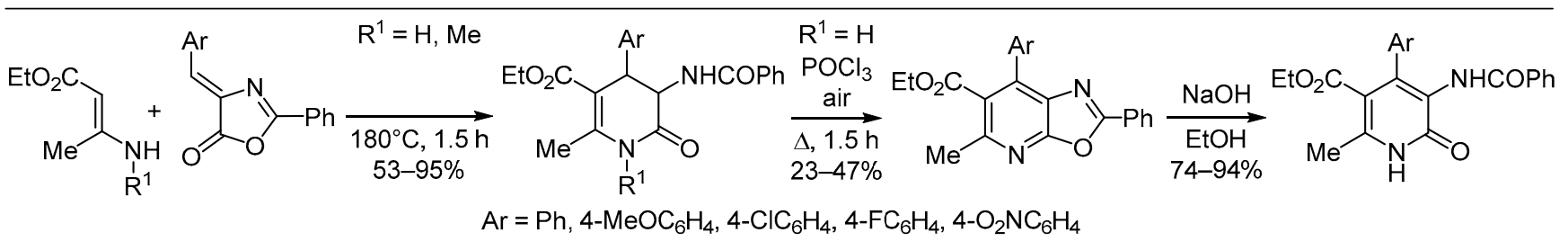

The reaction of 4-arylidene-2-phenyloxazol-5(4H)-ones with enamines of ethyl acetoacetate gave 4-aryl-2-methyl-6-oxo5-[(phenylcarbonyl)amino]-1,4,5,6-tetrahydropyridine-3-carboxylic acid esters, which, when heated with phosphorus oxychloride, were converted into esters of 7-aryl-5-methyl-2-phenyloxazolo[5,4-b]pyridine-6-carboxylic acids. Alkaline hydrolysis of these compounds gave 4-aryl-2-methyl-6-oxo-5-[(phenylcarbonyl)amino]-1,6-dihydropyridine-3-carboxylic acid esters.

Keywords: 3 -amino-4-arylpyridin-2(1H)-ones, azlactones, oxazolo[5,4-b]pyridines, alkaline hydrolysis, oxidation.

Pyridin-2(1H)-ones are privileged structures that are part of many biologically active compounds of natural and synthetic origin. ${ }^{1} \quad 3$-Aminopyridin-2(1H)-ones are of particular interest since they contain an amino acid amide fragment in their structure and can be used for the synthesis of peptidomimetics. Among these compounds, enzyme inhibitors $^{2-6}$ such as $\mathrm{M}^{\text {pro }}$ protease which prevents the replication of the coronavirus SARS-CoV-2, ${ }^{7,8}$ agonists and modulators of cannabinoid receptors $\mathrm{CB} 2,{ }^{9,10}$ antagonists of prostaglandin receptor EP3, ${ }^{11}$ and an inhibitor of jasmonate signaling ${ }^{12}$ have been found. 5-Amino-3,4'-bipyridin$(1 H)$-one, known as the cardiotonic drug amrinone, ${ }^{13}$ is widely used in clinical practice. 3-Aminopyridin-2(1H)ones are used in the synthesis of more complex compounds. ${ }^{14-16}$ Recently, it was shown that 3-amino4-arylpyridin-2(1H)-ones are good antioxidants, ${ }^{17,18}$ possess luminescent properties, ${ }^{19}$ and can be used as luminescent dyes for enzyme-linked immunosorbent assay. ${ }^{20}$ Despite the wide range of biological activity, the methods for the preparation of 3-amino-4-arylpyridin$2(1 H)$-ones are few and, as a rule, involve multistep synthesis. ${ }^{19-22}$
It was previously reported that azlactones are capable of reacting with enamines of 1,3-diketones and 1,3-keto esters to form the corresponding amides of 3-amino-4-aryl-3,4-dihydropyridin-2(1H)-ones, ${ }^{23-25}$ which, in principle, can be oxidized to the corresponding amides of 3-aminopyridin2(1H)-ones. ${ }^{26-28}$ Therefore, it seemed necessary to study the possibility of such an approach to the synthesis of 3-amino-4-arylpyridin-2(1H)-one derivatives.

For this purpose, azlactones 1a-e were synthesized by the known method ${ }^{29}$ by condensation of hippuric acid with aromatic aldehydes in the presence of polyphosphoric acid. Compounds 1a-e obtained in this way were subjected to a reaction with enamines 2,3 by heating at $180^{\circ} \mathrm{C}$ for $1.5 \mathrm{~h}$ without a solvent. As a result, ethyl 4-aryl-2-methyl6-oxo-5-[(phenylcarbonyl)amino]-1,4,5,6-tetrahydropyridine3-carboxylates $\mathbf{4 a}-\mathbf{f}$ were obtained. Compounds $\mathbf{4 a}-\mathbf{c}$ were isolated as cis-isomers, while compounds $\mathbf{4 d}-\mathbf{f}$ represented mixtures of cis-/trans-isomers with yields of $64 / 22 \%$ (compound 4d), 79/16\% (compound 4e), 47/18\% (compound 4f); the mixtures were then resolved by silica gel column chromatography. It should be noted that the yields of dihydropyridones $\mathbf{4 c}-\mathbf{e}$ containing acceptor 
substituents in the aryl fragment were higher than those of unsubstituted and methoxy-substituted products $\mathbf{4 a , b}$ (Scheme 1, Table 1).

\section{Scheme 1}

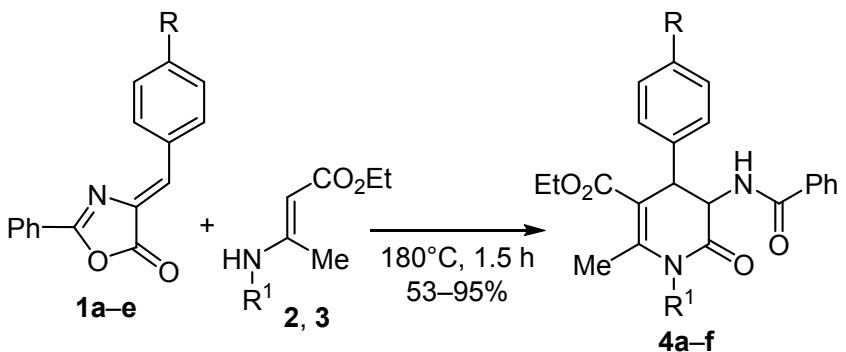

$$
\begin{aligned}
& 1 \text { a } \mathrm{R}=\mathrm{H}, \mathbf{b} \mathrm{R}=\mathrm{OMe}, \mathbf{c} \mathrm{R}=\mathrm{Cl}, \mathbf{d} \mathrm{R}=\mathrm{F}, \text { e } \mathrm{R}=\mathrm{NO}_{2} \\
& 2 \mathrm{R}^{1}=\mathrm{H} ; 3 \mathrm{R}^{1}=\mathrm{Me} \\
& 4 \text { a } \mathrm{R}=\mathrm{H}, \mathrm{R}^{1}=\mathrm{H} \text {; b R = OMe, } \mathrm{R}^{1}=\mathrm{H} ; \mathbf{c} \mathrm{R}=\mathrm{Cl}, \mathrm{R}^{1}=\mathrm{H} \\
& \text { d } R=F, R^{1}=H ; \text { e } R=N_{2} ; R^{1}=H ; f R=H, R^{1}=M e
\end{aligned}
$$

Table 1. Yields of isomers of compounds $4 a-f$

\begin{tabular}{ccc}
\hline \multirow{2}{*}{ Compound } & \multicolumn{2}{c}{ Yield, \% } \\
\cline { 2 - 3 } & cis-Isomer & trans-Isomer \\
\hline $\mathbf{4 a}$ & 58 & - \\
$\mathbf{4 b}$ & 53 & - \\
$\mathbf{4 c}$ & 74 & - \\
$\mathbf{4 d}$ & 64 & 22 \\
$\mathbf{4 e}$ & 79 & 16 \\
$\mathbf{4 f}$ & 47 & 18 \\
\hline
\end{tabular}

The structure and composition of the obtained compounds were confirmed by the data of IR, ${ }^{1} \mathrm{H}$ and ${ }^{13} \mathrm{C}$ NMR spectroscopy, X-ray structural analysis, and elemental analysis. In the ${ }^{1} \mathrm{H}$ NMR spectra of cis-isomers 4a-e, the protons at position $\mathrm{C}-4$ of the pyridone ring are present in the form of doublets, whereas at position C-5 as doublets of doublets with ${ }^{3} J_{5-\mathrm{CH}, \mathrm{NH}}=5.3-6.7 \mathrm{~Hz}$ and total coupling constant ${ }^{3} J_{4-\mathrm{CH}, 5-\mathrm{CH}}=7.4-8.0 \mathrm{~Hz}$. Due to the low solubility of trans-isomers $\mathbf{4 d , e}$ in $\mathrm{CDCl}_{3}$, their ${ }^{1} \mathrm{H}$ NMR spectra were recorded in DMSO- $d_{6}$.

According to X-ray diffraction data, the crystal of compound 4a consists of molecules of only one pair of enantiomers with the cis arrangement of substituents and the $3 R^{*}, 4 R^{*}$ configuration of the atoms of the methine groups (the numbering of atoms is shown in Figure 1). The conformation of the pyridone ring can be described as a highly distorted boat. Atoms N(1), C(1), C(2), and C(3) lie in the same plane, atoms $\mathrm{C}(5)$ and $\mathrm{C}(4)$ deviate from this plane to one side by 0.15 and $0.67 \AA$, respectively. In the solid state, molecules are linked into infinite chains via $\mathrm{N}(1)-\mathrm{H}(1) \cdots \mathrm{O}(2)$ hydrogen bonds.

To oxidate obtained 3,4-dihydropyridin-2(1H)-ones, $\mathrm{MnO}_{2},{ }^{27,28} \mathrm{FeCl}_{3},{ }^{30}$ and $\mathrm{DDQ}^{31,32}$ were employed. However, in our case, oxidation with these reagents, chloranil, $\mathrm{NaNO}_{2}$ in $\mathrm{AcOH}, \mathrm{K}_{2} \mathrm{~S}_{2} \mathrm{O}_{8}$ in $\mathrm{MeCN}$, by heating compounds 4a,f with $\mathrm{KMnO}_{4}$ in $\mathrm{Me}_{2} \mathrm{CO}$ or heating with $10 \% \mathrm{Pd} / \mathrm{C}$ in

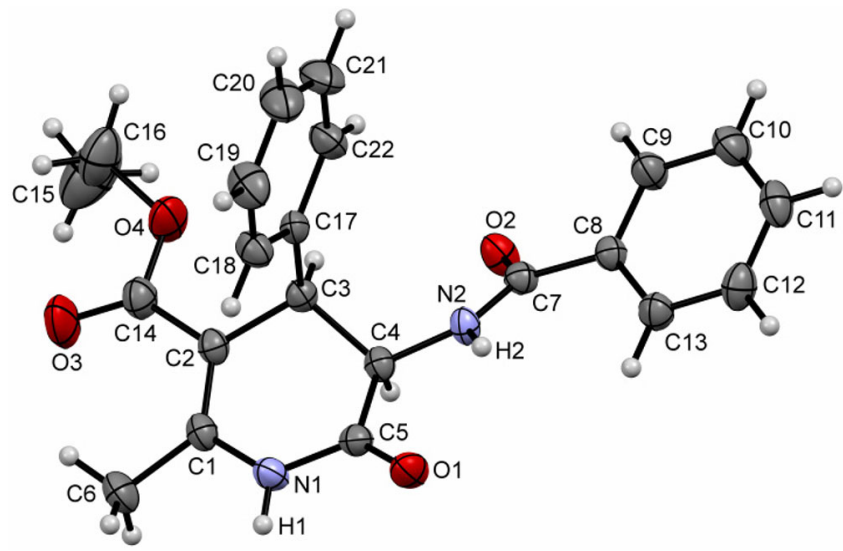

Figure 1. The molecular structure of compound $\mathbf{4 a}$ with atoms represented as thermal vibration ellipsoids with $30 \%$ probability.

xylene did not lead to the formation of pyridones 5a,f (Scheme 2).

Scheme 2<smiles>[R17]N1C(=O)C(NC(=O)c2ccccc2)C(c2ccccc2)C(C(=O)OCC)=C1C</smiles>

It is known that 1,4-dihydropyridines can be easily oxidized to pyridines. In some cases, oxidation with atmospheric oxygen occurs already upon the preparation of 1,4-dihydropyridines (Scheme 3). ${ }^{33} \mathrm{We}$ studied the possibility of obtaining 4,7-dihydrooxazolo[5,4-b]pyridine $\mathbf{6 a}$ by the action of the following dehydrating reagents on compound 4a (Scheme 3, Table 2): $\mathrm{POCl}_{3}, \mathrm{SOCl}_{2}$, polyphosphoric acid (PPA), $\mathrm{Ac}_{2} \mathrm{O}$ in the presence of $\mathrm{H}_{2} \mathrm{SO}_{4}$. Heating compound 4 a with $\mathrm{POCl}_{3}, \mathrm{SOCl}_{2}$, or $\mathrm{Ac}_{2} \mathrm{O}$

Scheme 3

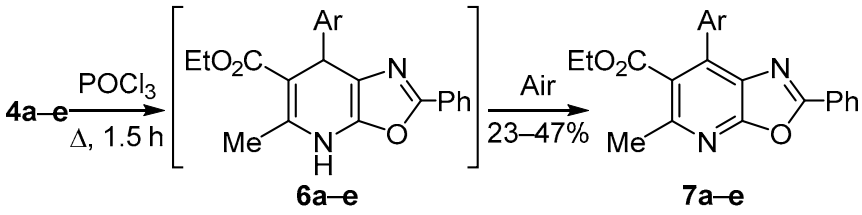

a Ar $=\mathrm{Ph}, \mathbf{b} \mathrm{Ar}=4-\mathrm{MeOC}_{6} \mathrm{H}_{4}, \mathbf{c ~ A r}=4-\mathrm{ClC}_{6} \mathrm{H}_{4}$, d $\mathrm{Ar}=4-\mathrm{FC}_{6} \mathrm{H}_{4}, \mathrm{e} \mathrm{Ar}=4-\mathrm{O}_{2} \mathrm{NC}_{6} \mathrm{H}_{4}$

Table 2. Yields of oxazolo[5,4-b]pyridine $7 \mathbf{a}$ by the action of dehydrating reagents on compound $\mathbf{4 a}$

\begin{tabular}{cccc}
\hline Reagent & Temperature, ${ }^{\circ} \mathrm{C}$ & Time, $\mathrm{h}$ & Yield, \% \\
\hline $\mathrm{POCl}_{3}$ & 25 & 24 & 0 \\
$\mathrm{POCl}_{3}$ & 110 & 1.5 & 40 \\
$\mathrm{SOCl}_{2}$ & 25 & 24 & 0 \\
$\mathrm{SOCl}_{2}$ & 75 & 3 & 34 \\
$\mathrm{Ac}_{2} \mathrm{O}, \mathrm{H}_{2} \mathrm{SO}_{4}$ & 140 & 17 & 10 \\
$\mathrm{PPA}$ & 150 & 10 & 0 \\
\hline
\end{tabular}


Table 3. The data of absorption and fluorescence spectra of compounds $7 \mathbf{a}-\mathbf{e}$ and $\mathbf{8 a}$

\begin{tabular}{|c|c|c|c|c|c|c|}
\hline \multirow{2}{*}{ Compound } & \multicolumn{2}{|c|}{ UV spectrum } & \multicolumn{4}{|c|}{ Photoluminescence } \\
\hline & ${ }^{\max } \lambda_{\mathrm{abs}}, \mathrm{nm}$ & $\varepsilon, 10^{3} \mathrm{M}^{-1} \cdot \mathrm{cm}^{-1}$ & $\lambda_{\mathrm{ex}}, \mathrm{nm}$ & ${ }^{\max } \lambda_{\mathrm{em}}, \mathrm{nm}$ & Stokes shift, eV; nm & Quantum yield, $\Phi_{\mathrm{f}}$ \\
\hline $7 \mathbf{a}$ & 256,313 & $31.0 \pm 0.5(\lambda 313 \mathrm{~nm})$ & 290,310 & 366 & $0.57 ; 53$ & $0.16 \pm 0.00$ \\
\hline $7 b$ & 266,314 & $31.9 \pm 0.6(\lambda 314 \mathrm{~nm})$ & 310,315 & 412 & $0.94 ; 98$ & $0.19 \pm 0.01$ \\
\hline $7 \mathrm{c}$ & 258,315 & $31.0 \pm 0.8(\lambda 315 \mathrm{~nm})$ & 310,315 & 371 & $0.59 ; 56$ & $0.19 \pm 0.01$ \\
\hline $7 d$ & 258,314 & $30.5 \pm 0.9(\lambda 314 \mathrm{~nm})$ & 310,315 & 366 & $0.56 ; 52$ & $0.14 \pm 0.01$ \\
\hline $7 e$ & 279,323 & $25.7 \pm 0.3(\lambda 323 \mathrm{~nm})$ & 315 & - & - & $0.00 \pm 0.00$ \\
\hline & 259,319 & $27.5 \pm 0.7(\lambda 319 \mathrm{~nm})$ & $310 ; 320$ & 364 & $0.48 ; 45$ & $0.82 \pm 0.03$ \\
\hline
\end{tabular}

in $\mathrm{H}_{2} \mathrm{SO}_{4}$ as a result of oxidation of intermediate $6 \mathbf{a}$ with atmospheric oxygen gave oxazolo[5,4-b]pyridine $7 \mathbf{a}$, isolated in 40, 34, and 10\% yields (Scheme 3, Table 2). The yields of oxazolo[5,4-b]pyridines $7 \mathbf{a}-\mathbf{e}$ obtained by heating compounds $4 \mathbf{a}-\mathbf{e}$ with $\mathrm{POCl}_{3}$ were $23-47 \%$.

Comparison of the electronic spectrum of compound $7 \mathbf{a}$ with the previously reported ${ }^{34}$ spectrum of oxazolo[5,4-b]pyridine 8a, which does not contain an ethoxycarbonyl group at the C-5 position of the heterocycle, showed that they differ little from each other (Table 3, Fig. 2). The introduction of an acceptor substituent into the pyridine ring results in a hypsochromic shift of both absorption and luminescence bands by $15-35 \mathrm{~nm}$ and a decrease in the luminescence quantum yield from 0.82 to 0.16 . The absorption spectra of oxazolo[5,4- $b]$ pyridines $7 \mathbf{a}-\mathbf{e}$ have two maxima centered at 256-279 and 313-323 nm. Compound 7e, containing a nitro group, does not luminesce, whereas compounds $\mathbf{7 a}, \mathbf{c}, \mathbf{d}$ contain an intense band at $366-371 \mathrm{~nm}$ in the luminescence spectrum. The introduction of electron-donating methoxy group into the aryl substituent (compound $\mathbf{7 b}$ ) results in a significant bathochromic shift. The center of the emission band in the spectrum of compound $\mathbf{7 b}$ is shifted by $46 \mathrm{~nm}$ compared with the spectrum of 5-methyl-2,7-diphenyloxazolo[5,4-b]pyridine-6-carboxylic acid ethyl ester (7a), while the Stokes shift increases from 0.57 (compound 7a) to $0.94 \mathrm{eV}$ (compound $\mathbf{7 b}$ ). The luminescence quantum yields of compounds 7a-d are 0.14-0.19.

Upon heating with an aqueous ethanol solution of $\mathrm{NaOH}$ for $1.5 \mathrm{~h}$, opening of the oxazole ring of oxazolo[5,4-b]pyridines $7 \mathbf{a}-\mathbf{e}$ takes place leading to amides $9 \mathbf{a}-\mathbf{e}$ in 74 $94 \%$ yields (Scheme 4 ).

\section{Scheme 4}<smiles>CCOC(=O)c1c(C)[nH]c(=O)c(NC(=O)c2ccccc2)c1Br</smiles>

$\mathbf{a} \mathrm{Ar}=\mathrm{Ph}, \mathbf{b} \mathrm{Ar}=4-\mathrm{MeOC}_{6} \mathrm{H}_{4}, \mathbf{c ~ A r}=4-\mathrm{ClC}_{6} \mathrm{H}_{4}$, d $\mathrm{Ar}=4-\mathrm{FC}_{6} \mathrm{H}_{4}$, e $\mathrm{Ar}=4-\mathrm{O}_{2} \mathrm{NC}_{6} \mathrm{H}_{4}$

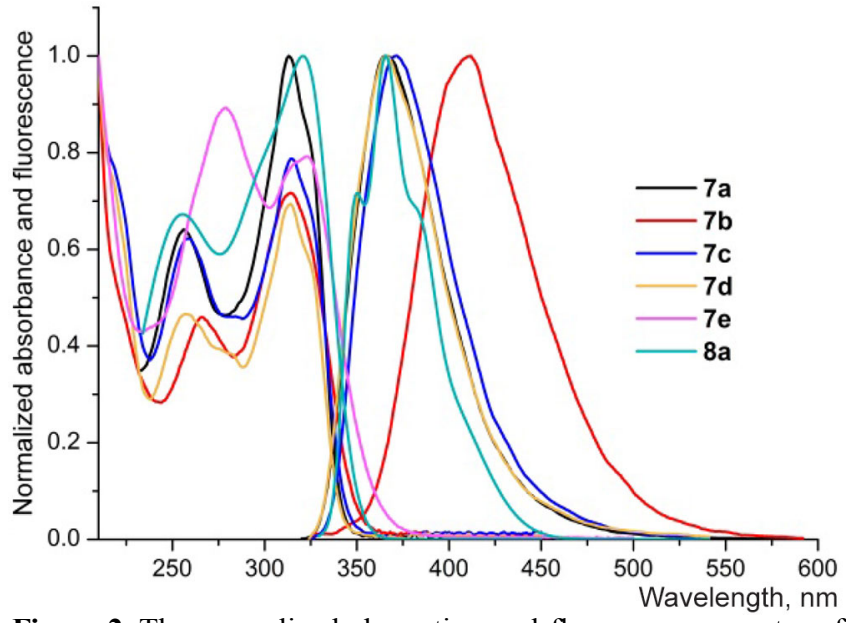

Figure 2. The normalized absorption and fluorescence spectra of compounds $7 \mathbf{a}-\mathbf{e}$ and $\mathbf{8 a}$ in $\mathrm{EtOH}$.

To conclude, we developed a simple method for the preparation of 7 -aryloxazolo $[5,4-b]$ pyridines based on the accessible azlactones and enamines of ethyl acetoacetate. The photophysical properties of these compounds were studied and it has been shown that their hydrolysis leads to 3 -aminopyridin-2(1H)-one derivatives in good yields.

\section{Experimental}

IR spectra were registered on a Simex FT-801 Fourier transform spectrometer in $\mathrm{KBr}$ pellets. Absorption spectra were recorded on a PerkinElmer Lambda 750 diode array spectrophotometer, photoluminescence spectra were recorded on a Cary Eclipse fluorescence spectrophotometer. In both cases, the test compounds were dissolved in $\mathrm{EtOH}$ so that the concentration of the resulting solutions was lower than $10^{-5} \mathrm{~mol} / \mathrm{dm}^{3}$. The molar light absorption coefficient was determined according to the described method. ${ }^{35}$ The quantum yield was determined relative to quinine sulfate in $0.5 \mathrm{M} \mathrm{H}_{2} \mathrm{SO}_{4}\left(\Phi_{\mathrm{f}} 0.546\right)$ using the comparison method. ${ }^{36}{ }^{1} \mathrm{H}$ and ${ }^{13} \mathrm{C}$ NMR spectra were acquired on a Bruker DRX-400 spectrometer (400 and $100 \mathrm{MHz}$, respectively) in $\mathrm{CDCl}_{3}$ or DMSO- $d_{6}$, with the residual solvent signals $\left(\mathrm{CDCl}_{3}: 7.26 \mathrm{ppm}\right.$ for ${ }^{1} \mathrm{H}$ nuclei and $77.0 \mathrm{ppm}$ for ${ }^{13} \mathrm{C}$ nuclei; DMSO- $d_{6}: 2.50 \mathrm{ppm}$ for ${ }^{1} \mathrm{H}$ 
nuclei and $39.5 \mathrm{ppm}$ for ${ }^{13} \mathrm{C}$ nuclei) serving as internal standards. Elemental analysis was performed on a Carlo Erba EA 1106 CHN-analyzer. Melting points were determined on a Reach Devices RD-MP apparatus and are uncorrected. Monitoring of the reaction progress and assessment of the purity of synthesized compounds were done by TLC on Silufol UV-254 plates, eluents $\mathrm{CHCl}_{3}$ and $\mathrm{CHCl}_{3}$-EtOAc, 10:1. Visualization of plates was done under UV light. Silica gel 60 (0.063-0.200 mm, MachereyNagel) was used for column chromatography.

The starting azlactones 1a-e were obtained according to a known procedure ${ }^{29}$ by condensation of hippuric acid with aromatic aldehydes in the presence of polyphosphoric acid. The starting enamines of acetoacetic ester $\mathbf{2}, \mathbf{3}$ were obtained by the previously described methods. ${ }^{37}$

Synthesis of 1,4,5,6-tetrahydropyridin-6-ones 4a-f (General method). A mixture of azlactone 1a-e $(10 \mathrm{mmol})$ and enamine 2 or $3(10 \mathrm{mmol})$ was heated at $180^{\circ} \mathrm{C}$ for $1.5 \mathrm{~h}$. The mixture was then cooled and purified by column chromatography on silica gel, eluent $\mathrm{CHCl}_{3}$-EtOAc, 10:1.

Ethyl cis-2-methyl-6-oxo-4-phenyl-5-[(phenylcarbonyl)amino]-1,4,5,6-tetrahydropyridine-3-carboxylate (cis-4a). Yield $2190 \mathrm{mg}(58 \%)$, colorless crystals, $\mathrm{mp} 205-206^{\circ} \mathrm{C}$ $(\mathrm{EtOH})\left(\mathrm{mp} 200-201{ }^{\circ} \mathrm{C}(\mathrm{PhH})^{24}\right)$. IR spectrum, $v, \mathrm{~cm}^{-1}$ : 3397, 3337, 3165, 3031, 2992, 2979, 2953, 2904, 1701, $1645,1634,1602,1580,1528,1490,1454,1401,1295$, 1255, 1226, 1193, 1171, 1082, 941, 734, 716, 697, 647. ${ }^{1} \mathrm{H}$ NMR spectrum $\left(\mathrm{CDCl}_{3}\right), \delta, \mathrm{ppm}(J, \mathrm{~Hz}): 1.12(3 \mathrm{H}, \mathrm{t}$, $\left.J=7.0, \mathrm{CH}_{2} \mathrm{CH}_{3}\right) ; 2.46\left(3 \mathrm{H}, \mathrm{s}, 2-\mathrm{CH}_{3}\right) ; 3.99-4.12(2 \mathrm{H}, \mathrm{m}$, $\left.\mathrm{CH}_{2} \mathrm{CH}_{3}\right) ; 4.71(1 \mathrm{H}, \mathrm{d}, J=7.8,4-\mathrm{CH}) ; 5.21(1 \mathrm{H}, \mathrm{dd}$, $J=7.8, J=6.7,5-\mathrm{CH}) ; 6.46(1 \mathrm{H}, \mathrm{d}, J=6.7,5-\mathrm{N} \underline{\mathrm{HCOPh}})$; 7.08-7.11 (2H, m, H Ph); 7.23-7.25 (3H, m, H Ph); 7.36$7.40(2 \mathrm{H}, \mathrm{m}, \mathrm{H} \mathrm{Ph}) ; 7.46-7.50(1 \mathrm{H}, \mathrm{m}, \mathrm{H} \mathrm{Ph}) ; 7.62-7.64$ $(2 \mathrm{H}, \mathrm{m}, \mathrm{H} \mathrm{Ph}) ; 7.99(1 \mathrm{H}, \mathrm{s}, 1-\mathrm{NH}) .{ }^{13} \mathrm{C}$ NMR spectrum $\left(\mathrm{CDCl}_{3}\right), \delta$, ppm: $14.0 ; 18.8 ; 42.3 ; 53.4 ; 60.4 ; 108.9 ; 127.0$; $127.7 ; 128.3 ; 128.6 ; 128.7 ; 131.8 ; 133.9 ; 136.8 ; 145.2$; 166.2; 167.4; 169.2. Found, \%: C 69.96; H 6.03; N 7.46. $\mathrm{C}_{22} \mathrm{H}_{22} \mathrm{~N}_{2} \mathrm{O}_{4}$. Calculated, \%: C 69.83; H 5.86; $\mathrm{N} 7.40$.

Ethyl cis-4-(4-methoxyphenyl)-2-methyl-6-oxo-5-[(phenylcarbonyl)amino]-1,4,5,6-tetrahydropyridine-3-carboxylate (cis-4b). Yield $2160 \mathrm{mg}$ (53\%), beige crystals, mp 161$162^{\circ} \mathrm{C}(i-\mathrm{PrOH})\left(\mathrm{mp} 212-214^{\circ} \mathrm{C}(\mathrm{PhH})^{24}\right)$. IR spectrum, $v, \mathrm{~cm}^{-1}: 3397,3232,3156,3067,2982,2955,2938,2906$, $2838,1701,1637,1612,1581,1529,1512,1489,1465$, $1445,1399,1385,1374,1288,1249,1206,1177,1091$, $1030,831,765,712,693,647 .{ }^{1} \mathrm{H}$ NMR spectrum $\left(\mathrm{CDCl}_{3}\right)$, $\delta, \operatorname{ppm}(J, \mathrm{~Hz}): 1.14\left(3 \mathrm{H}, \mathrm{t}, J=7.1, \mathrm{CH}_{2} \underline{\mathrm{CH}}_{3}\right) ; 2.45(3 \mathrm{H}, \mathrm{s}$, $\left.2-\mathrm{CH}_{3}\right) ; 3.74\left(3 \mathrm{H}, \mathrm{s}, \mathrm{OCH}_{3}\right) ; 3.99-4.13\left(2 \mathrm{H}, \mathrm{m}, \mathrm{CH}_{2} \mathrm{CH}_{3}\right)$; $4.65(1 \mathrm{H}, \mathrm{d}, J=7.8,4-\mathrm{CH}) ; 5.17(1 \mathrm{H}, \mathrm{dd}, J=7.8, J=6.7$, $5-\mathrm{CH}) ; 6.46(1 \mathrm{H}, \mathrm{d}, J=6.7,5-\mathrm{NHCOPh}) ; 6.75-6.79(2 \mathrm{H}$, $\mathrm{m}, \mathrm{H}-3,5 \mathrm{Ar}) ; 7.00-7.03(2 \mathrm{H}, \mathrm{m}, \mathrm{H}-2,6 \mathrm{Ar}) ; 7.37-7.41(2 \mathrm{H}$, $\mathrm{m}, \mathrm{H} \mathrm{Ph}) ; 7.46-7.50(1 \mathrm{H}, \mathrm{m}, \mathrm{H} \mathrm{Ph}) ; 7.64-7.67(2 \mathrm{H}, \mathrm{m}$, $\mathrm{H} \mathrm{Ph}) ; 7.98(1 \mathrm{H}$, br. s, $1-\mathrm{NH}) .{ }^{13} \mathrm{C}$ NMR spectrum $\left(\mathrm{CDCl}_{3}\right)$, $\delta$, ppm: $14.0 ; 18.8 ; 41.5 ; 53.6 ; 55.2 ; 60.3 ; 109.2 ; 114.0$; $127.0 ; 128.6(2 \mathrm{C}) ; 129.3 ; 131.8 ; 133.9 ; 144.9 ; 159.1$; 166.3 ; 167.4; 169.3. Found, \%: C 67.50; H 5.98; N 6.92. $\mathrm{C}_{23} \mathrm{H}_{24} \mathrm{~N}_{2} \mathrm{O}_{5}$. Calculated, \%: C 67.63; H 5.92; N 6.86 .

Ethyl cis-4-(4-chlorophenyl)-2-methyl-6-oxo-5-[(phenylcarbonyl)amino]-1,4,5,6-tetrahydropyridine-3-carboxylate (cis-4c). Yield $3060 \mathrm{mg}$ (74\%), colorless crystals, mp 197$198^{\circ} \mathrm{C}(i-\mathrm{PrOH})$. IR spectrum, $v, \mathrm{~cm}^{-1}: 3416,3235,3133$, 2985, 2962, 2902, 1702, 1660, 1635, 1580, 1517, 1487, 1394, 1298, 1270, 1228, 1184, 1103, 1085, 1014, 832, 790, $717,668 .{ }^{1} \mathrm{H}$ NMR spectrum $\left(\mathrm{CDCl}_{3}\right), \delta$, ppm $(J, \mathrm{~Hz}): 1.13$ $\left(3 \mathrm{H}, \mathrm{t}, J=7.0, \mathrm{CH}_{2} \mathrm{CH}_{3}\right) ; 2.47\left(3 \mathrm{H}, \mathrm{s}, 2-\mathrm{CH}_{3}\right) ; 4.01-4.12$ $\left(2 \mathrm{H}, \mathrm{m}, \mathrm{CH}_{2} \mathrm{CH}_{3}\right) ; 4.73(1 \mathrm{H}, \mathrm{d}, J=7.8,4-\mathrm{CH}) ; 5.15(1 \mathrm{H}$, $\mathrm{dd}, J=7.8, J=6.0,5-\mathrm{CH}) ; 6.56(1 \mathrm{H}, \mathrm{d}, J=6.0$, 5-NHCOPh); 7.00-7.03 (2H, m, H-2,6 Ar); 7.18-7.22 (2H, m, H-3,5 Ar); 7.38-7.43 (2H, m, H Ph); 7.48-7.52 (1H, m, $\mathrm{H} \mathrm{Ph}) ; 7.64-7.67$ (2H, m, H Ph); 8.04 (1H, br. s, 1-NH). ${ }^{13} \mathrm{C}$ NMR spectrum $\left(\mathrm{CDCl}_{3}\right), \delta$, ppm: $14.0 ; 18.8 ; 41.5$; $53.4 ; 60.5 ; 108.6 ; 127.0 ; 128.7 ; 128.8 ; 129.6 ; 132.0 ; 133.5$; $133.6 ; 135.3 ; 145.4 ; 166.0 ; 167.5 ; 169.0$. Found, \%: C 63.81; H 5.18; N 6.74. $\mathrm{C}_{22} \mathrm{H}_{21} \mathrm{ClN}_{2} \mathrm{O}_{4}$. Calculated, \%: C 64.00; H 5.13; N 6.79.

Ethyl cis-4-(4-fluorophenyl)-2-methyl-6-oxo-5-[(phenylcarbonyl)amino]-1,4,5,6-tetrahydropyridine-3-carboxylate (cis-4d). Yield $2530 \mathrm{mg}$ (64\%), colorless crystals, mp 180 $181^{\circ} \mathrm{C}(i-\mathrm{PrOH})$. IR spectrum, $v, \mathrm{~cm}^{-1}: 3408,3246,3135$, 2979, 2874, 1707, 1634, 1604, 1581, 1510, 1486, 1387, 1299, 1230, 1160, 1097, 1083, 941, 837, 786, 718, 694. ${ }^{1} \mathrm{H}$ NMR spectrum $\left(\mathrm{CDCl}_{3}\right), \delta$, ppm $(J, \mathrm{~Hz}): 1.12(3 \mathrm{H}, \mathrm{t}$, $\left.J=7.1, \mathrm{CH}_{2} \mathrm{CH}_{3}\right) ; 2.47\left(3 \mathrm{H}, \mathrm{s}, 2-\mathrm{CH}_{3}\right) ; 4.02-4.10(2 \mathrm{H}, \mathrm{m}$, $\left.\mathrm{CH}_{2} \mathrm{CH}_{3}\right) ; 4.74(1 \mathrm{H}, \mathrm{d}, J=7.8,4-\mathrm{CH}) ; 5.15(1 \mathrm{H}, \mathrm{dd}$, $J=7.8, J=6.0,5-\mathrm{CH}) ; 6.53(1 \mathrm{H}, \mathrm{d}, J=6.0,5-\mathrm{NHCOPh}$; 6.89-6.95 (2H, m, H-3,5 Ar); 7.03-7.08 (2H, m, H-2,6 Ar); 7.38-7.42 (2H, m, H Ph); 7.48-7.52 (1H, m, H Ph); 7.63$7.66(2 \mathrm{H}, \mathrm{m}, \mathrm{H} \mathrm{Ph}) ; 8.16(1 \mathrm{H}$, br. s, $1-\mathrm{NH}) .{ }^{13} \mathrm{C} \mathrm{NMR}$ spectrum $\left(\mathrm{CDCl}_{3}\right), \delta$, ppm $(J, \mathrm{~Hz}): 14.0 ; 18.8 ; 41.4 ; 53.5$; $60.4 ; 108.9 ; 115.5(\mathrm{~d}, J=21.7) ; 127.0 ; 128.6 ; 129.8(\mathrm{~d}$, $J=7.8) ; 131.9 ; 132.5$ (d, $J=3.5) ; 133.7 ; 145.2 ; 162.3$ (d, $J=246.2) ; 166.1 ; 167.5 ; 169.1$. Found, \%: C 66.86; $\mathrm{H}$ 5.47; N 7.15. $\mathrm{C}_{22} \mathrm{H}_{21} \mathrm{FN}_{2} \mathrm{O}_{4}$. Calculated, \%: C 66.66; H 5.34; N 7.07.

Ethyl trans-4-(4-fluorophenyl)-2-methyl-6-oxo-5-[(phenylcarbonyl)amino]-1,4,5,6-tetrahydropyridine-3-carboxylate

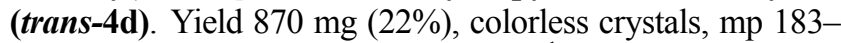
$184^{\circ} \mathrm{C}\left(i\right.$-PrOH). IR spectrum, $v, \mathrm{~cm}^{-1}: 3240,3160,3069$, 2979, 2962, 2931, 1709, 1644, 1604, 1580, 1537, 1511, 1492, 1366, 1275, 1226, 1192, 1124, 1097, 1016, 833, 693. ${ }^{1} \mathrm{H}$ NMR spectrum (DMSO- $\left.d_{6}\right), \delta, \mathrm{ppm}(J, \mathrm{~Hz}): 0.88(3 \mathrm{H}, \mathrm{t}$, $\left.J=7.0, \mathrm{CH}_{2} \mathrm{CH}_{3}\right) ; 2.30\left(3 \mathrm{H}, \mathrm{s}, 2-\mathrm{CH}_{3}\right) ; 3.80-3.91(2 \mathrm{H}, \mathrm{m}$, $\left.\mathrm{CH}_{2} \mathrm{CH}_{3}\right) ; 4.19(1 \mathrm{H}, \mathrm{d}, J=6.7,4-\mathrm{CH}) ; 4.59(1 \mathrm{H}, \mathrm{dd}$, $J=8.0, J=6.7,5-\mathrm{CH}) ; 7.10-7.14(2 \mathrm{H}, \mathrm{m}, \mathrm{H}-3,5 \mathrm{Ar}) ; 7.21-$ $7.25(2 \mathrm{H}, \mathrm{m}, \mathrm{H}-2,6 \mathrm{Ar}) ; 7.43-7.54(3 \mathrm{H}, \mathrm{m}, \mathrm{H} \mathrm{Ph}) ; 7.79$ $(2 \mathrm{H}, \mathrm{d}, J=7.4, \mathrm{H} \mathrm{Ph}) ; 8.91(1 \mathrm{H}, \mathrm{d}, J=8.0,5-\mathrm{NHCOPh})$; $10.15(1 \mathrm{H}, \mathrm{s}, 1-\mathrm{NH}) .{ }^{13} \mathrm{C}$ NMR spectrum (DMSO- $\left.d_{6}\right)$, $\delta$, ppm $(J, \mathrm{~Hz}): 13.7 ; 17.9 ; 44.8 ; 55.1 ; 59.3 ; 104.3 ; 115.2$ $(\mathrm{d}, J=21.7) ; 127.5 ; 128.2 ; 129.0(\mathrm{~d}, J=7.8) ; 131.4 ; 133.9$; 137.1 (d, $J=2.6) ; 146.2 ; 161.0(\mathrm{~d}, J=242.7) ; 166.5(2 \mathrm{C})$; 167.1. Found, \%: C 66.87; H 5.46; N 7.15. $\mathrm{C}_{22} \mathrm{H}_{21} \mathrm{FN}_{2} \mathrm{O}_{4}$. Calculated, \%: C 66.66; H 5.34; N 7.07.

Ethyl cis-2-methyl-4-(4-nitrophenyl)-6-oxo-5-[(phenylcarbonyl)amino]-1,4,5,6-tetrahydropyridine-3-carboxylate (cis-4e). Yield $3340 \mathrm{mg}$ (79\%), light-yellow crystals, $\mathrm{mp} \quad 201-202^{\circ} \mathrm{C} \quad(i-\mathrm{PrOH}) \quad\left(\mathrm{mp} \quad 201-202^{\circ} \mathrm{C} \quad(\mathrm{PhH})^{24}\right)$. IR spectrum, $v, \mathrm{~cm}^{-1}: 3399,3238,3126,2977,2869,1707$, $1634,1603,1581,1521,1486,1446,1388,1349,1298$, 
1233, 1212, 1169, 1084, 939, 855, 789, 719, 694. ${ }^{1} \mathrm{H}$ NMR spectrum $\left(\mathrm{CDCl}_{3}\right), \delta$, ppm $(J, \mathrm{~Hz}): 1.12(3 \mathrm{H}, \mathrm{t}, J=7.1$, $\left.\mathrm{CH}_{2} \mathrm{CH}_{3}\right) ; 2.51 \quad\left(3 \mathrm{H}, \quad \mathrm{s}, \quad 2-\mathrm{CH}_{3}\right) ; 4.00-4.13(2 \mathrm{H}, \mathrm{m}$, $\left.\mathrm{CH}_{2} \mathrm{CH}_{3}\right) ; 4.95(1 \mathrm{H}, \mathrm{d}, J=8.0,4-\mathrm{CH}) ; 5.16(1 \mathrm{H}, \mathrm{dd}$, $J=8.0, J=5.3,5-\mathrm{CH}) ; 6.64(1 \mathrm{H}, \mathrm{d}, J=5.3,5-\mathrm{NHCOPh})$; 7.24-7.27 (2H, m, H-2,6 Ar); 7.39-7.42 (2H, m, H Ph); 7.49$7.53(1 \mathrm{H}, \mathrm{m}, \mathrm{H} \mathrm{Ph}) ; 7.63-7.65(2 \mathrm{H}, \mathrm{m}, \mathrm{H} \mathrm{Ph}) ; 8.02(1 \mathrm{H}, \mathrm{s}$, 1-NH); 8.06-8.10 (2H, m, H-3,5 Ar). ${ }^{13} \mathrm{C}$ NMR spectrum $\left(\mathrm{CDCl}_{3}\right), \delta$, ppm: $14.0 ; 18.9 ; 41.9 ; 53.4 ; 60.7 ; 108.0 ; 123.8$; $127.0 ; 128.8 ; 129.2 ; 132.2 ; 133.2 ; 144.8 ; 145.8 ; 147.5$; 165.8; 167.6; 168.4. Found, \%: C 62.80; H 5.12; N 9.97. $\mathrm{C}_{22} \mathrm{H}_{21} \mathrm{~N}_{3} \mathrm{O}_{6}$. Calculated, \%: C 62.41; H 5.00; N 9.92.

Ethyl trans-2-methyl-4-(4-nitrophenyl)-6-0xo-5-[(phenylcarbonyl)amino]-1,4,5,6-tetrahydropyridine-3-carboxylate (trans-4e). Yield $677 \mathrm{mg}$ (16\%), light-yellow crystals, $\mathrm{mp} 157-158^{\circ} \mathrm{C}(i-\mathrm{PrOH})$. IR spectrum, $v, \mathrm{~cm}^{-1}: 3243$, $3163,3075,2979,1711,1645,1603,1581,1519,1490$, 1349, 1267, 1195, 1103, 1080, 1014, 841, 701. ${ }^{1} \mathrm{H}$ NMR spectrum (DMSO- $\left.d_{6}\right), \delta$, ppm $(J, \mathrm{~Hz}): 0.82(3 \mathrm{H}, \mathrm{t}, J=7.0$, $\left.\mathrm{CH}_{2} \mathrm{CH}_{3}\right) ; 2.31\left(3 \mathrm{H}, \quad \mathrm{s}, 2-\mathrm{CH}_{3}\right) ; 3.79-3.85(2 \mathrm{H}, \mathrm{m}$, $\left.\mathrm{CH}_{2} \mathrm{CH}_{3}\right) ; 4.35(1 \mathrm{H}, \mathrm{d}, J=6.7,4-\mathrm{CH}) ; 4.65(1 \mathrm{H}, \mathrm{dd}$, $J=7.1, J=6.7,5-\mathrm{CH}) ; 7.43-7.57(5 \mathrm{H}, \mathrm{m}, \mathrm{H} \mathrm{Ph}) ; 7.72-$ 7.83 (2H, m, H-2,6 Ar); 8.11-8.22 (2H, m, H-3,5 Ar); 8.93 $(1 \mathrm{H}, \mathrm{d}, J=7.1,5-\mathrm{NHCOPh}) ; 10.24(1 \mathrm{H}, \mathrm{s}, 1-\mathrm{NH})$. ${ }^{13} \mathrm{C}$ NMR spectrum (DMSO- $d_{6}$ ), $\delta$, ppm: $13.6 ; 17.9 ; 45.6$; 54.6 ; $59.4 ; 103.5 ; 123.7 ; 127.4 ; 128.3 ; 128.6 ; 131.5 ; 133.7$; $146.4 ; 146.9 ; 149.7 ; 166.2 ; 166.5 ; 166.8$. Found, \%: C $62.82 ; \mathrm{H} 5.10 ; \mathrm{N}$ 9.98. $\mathrm{C}_{22} \mathrm{H}_{21} \mathrm{~N}_{3} \mathrm{O}_{6}$. Calculated, \%: C $62.41 ;$ H $5.00 ;$ N 9.92.

Ethyl cis-1,2-dimethyl-6-oxo-4-phenyl-5-[(phenylcarbonyl)amino]-1,4,5,6-tetrahydropyridine-3-carboxylate (cis-4f). Yield $1840 \mathrm{mg}$ (47\%), beige crystals, mp 127$128^{\circ} \mathrm{C}(i-\mathrm{PrOH})$. IR spectrum, $v, \mathrm{~cm}^{-1}: 3389,3070,3034$, 2979, 2931, 2849, 1687, 1651, 1627, 1601, 1579, 1521, $1486,1383,1318,1291,1259,1240,1193,1177,1115$, 1089, 1037, 919, 843, 803, 788, 765, 714, 589. ${ }^{1} \mathrm{H}$ NMR spectrum $\left(\mathrm{CDCl}_{3}\right), \delta$, ppm $(J, \mathrm{~Hz}): 1.13(3 \mathrm{H}, \mathrm{t}, J=7.1$, $\left.\mathrm{CH}_{2} \mathrm{CH}_{3}\right) ; 2.62\left(3 \mathrm{H}, \mathrm{s}, 2-\mathrm{CH}_{3}\right) ; 3.31\left(3 \mathrm{H}, \mathrm{s}, 1-\mathrm{NCH}_{3}\right) ; 4.01$ $-4.15\left(2 \mathrm{H}, \mathrm{m}, \mathrm{CH}_{2} \mathrm{CH}_{3}\right) ; 4.73(1 \mathrm{H}, \mathrm{d}, J=7.4,4-\mathrm{CH}) ; 5.07$ $(1 \mathrm{H}, \mathrm{dd}, J=7.4, J=5.7,5-\mathrm{CH}) ; 6.67(1 \mathrm{H}, \mathrm{d}, J=5.7$, 5-NHCOPh); 6.94-6.99 (2H, m, H Ph); 7.20-7.24 (3H, m, $\mathrm{H} \mathrm{Ph})$; 7.36-7.41 (2H, m, H Ph); 7.46-7.51 (1H, m, H Ph); 7.65-7.68 $(2 \mathrm{H}, \mathrm{m}, \mathrm{H} \mathrm{Ph}) .{ }^{13} \mathrm{C}$ NMR spectrum $\left(\mathrm{CDCl}_{3}\right)$, $\delta$, ppm: $14.0 ; 16.9 ; 30.0 ; 41.2 ; 53.6 ; 60.5 ; 112.2 ; 127.0$; $127.6 ; 128.2 ; 128.5 ; 128.6 ; 131.7 ; 134.1 ; 136.0 ; 148.4$; 166.5; 167.2; 168.4. Found, \%: C 70.76; H 5.97; N 6.69. $\mathrm{C}_{23} \mathrm{H}_{24} \mathrm{~N}_{2} \mathrm{O}_{4}$. Calculated, \%: C 70.39; H 6.16; $\mathrm{N}$ 7.14.

Ethyl trans-1,2-dimethyl-6-oxo-4-phenyl-5-[(phenylcarbonyl)amino]-1,4,5,6-tetrahydropyridine-3-carboxylate (trans-4f). Yield $706 \mathrm{mg}(18 \%)$, beige crystals, mp 187$188^{\circ} \mathrm{C}\left(i\right.$-PrOH). IR spectrum, $v, \mathrm{~cm}^{-1}: 3311,3061,3035$, 2962, 2920, 2850, 1695, 1637, 1602, 1578, 1547, 1491, $1455,1365,1332,1288,1273,1203,1177,1123,1105$, 1093, 1075, 1053, 1006, 904, 860, 799, 754, 719, 701, 693, 692, 586. ${ }^{1} \mathrm{H}$ NMR spectrum $\left(\mathrm{CDCl}_{3}\right), \delta, \operatorname{ppm}(J, \mathrm{~Hz}): 0.87$ $\left(3 \mathrm{H}, \mathrm{t}, J=7.0, \mathrm{CH}_{2} \mathrm{CH}_{3}\right) ; 2.43\left(3 \mathrm{H}, \mathrm{d}, J=1.6,2-\mathrm{CH}_{3}\right) ; 3.18$ $\left(3 \mathrm{H}, \mathrm{s}, 1-\mathrm{NCH}_{3}\right) ; 3.79-3.89\left(2 \mathrm{H}, \mathrm{m}, \mathrm{CH}_{2} \mathrm{CH}_{3}\right) ; 4.19(1 \mathrm{H}$, $\mathrm{dd}, J=7.6, J=1.6,4-\mathrm{CH}) ; 4.96(1 \mathrm{H}, \mathrm{dd}, J=8.4, J=7.6$, $5-\mathrm{CH}) ; 6.38(1 \mathrm{H}, \mathrm{d}, J=8.4,5-\mathrm{NHCOPh}) ; 7.10-7.22(5 \mathrm{H}$, $\mathrm{m}, \mathrm{H} \mathrm{Ph}) ; 7.28-7.32(2 \mathrm{H}, \mathrm{m}, \mathrm{H} \mathrm{Ph}) ; 7.37-7.42(1 \mathrm{H}, \mathrm{m}$, $\mathrm{H} \mathrm{Ph}) ; 7.55-7.59(2 \mathrm{H}, \mathrm{m}, \mathrm{H} \mathrm{Ph}) .{ }^{13} \mathrm{C}$ NMR spectrum $\left(\mathrm{CDCl}_{3}\right), \delta$, ppm: $13.7 ; 16.7 ; 29.9 ; 45.6 ; 54.8 ; 60.5 ; 110.6$; $127.1 ; 127.4 ; 127.5 ; 128.5 ; 128.7 ; 131.7 ; 134.0 ; 138.5$; $145.9 ; 167.3$ (3C). Found, \%: C 70.75; H 5.99; N 6.73. $\mathrm{C}_{23} \mathrm{H}_{24} \mathrm{~N}_{2} \mathrm{O}_{4}$. Calculated, \%: C 70.39; H 6.16; N 7.14.

Synthesis of oxazolo[5,4-b]pyridines 7a-e (General method). A mixture of 1,4,5,6-tetrahydropyridin-6-one $\mathbf{4 a - e}$ $(1 \mathrm{mmol})$ and $\mathrm{POCl}_{3}(3 \mathrm{ml})$ was heated under reflux for $1.5 \mathrm{~h}$. The reaction mixture was evaporated to dryness, diluted with cold $\mathrm{H}_{2} \mathrm{O}(10 \mathrm{ml})$ and triturated to a homogeneous powder, which was filtered and washed with $\mathrm{H}_{2} \mathrm{O}(3 \times 5 \mathrm{ml})$. The product was purified by column chromatography on silica gel (eluent $\mathrm{CHCl}_{3}$ ) and recrystallized from $i$-PrOH.

Ethyl 5-methyl-2,7-diphenyloxazolo[5,4-b]pyridine6-carboxylate (7a). Yield $143 \mathrm{mg}(40 \%)$, colorless crystals, mp $145-146^{\circ} \mathrm{C}(i-\mathrm{PrOH})$. IR spectrum, $v, \mathrm{~cm}^{-1}$ : 3062 , 3056, 3034, 2980, 2941, 2929, 2904, 1720, 1623, $1602,1595,1573,1549,1495,1480,1451,1419,1380$, $1371,1361,1352,1316,1270,1253,1199,1184,1176$, $1158,1079,1048,1021,950,911,825,787,780,763,723$, 708, 690, 669, 651. ${ }^{1} \mathrm{H}$ NMR spectrum $\left(\mathrm{CDCl}_{3}\right), \delta$, ppm $(J, \mathrm{~Hz}): 1.01\left(3 \mathrm{H}, \mathrm{t}, J=7.2, \mathrm{CH}_{2} \mathrm{CH}_{3}\right) ; 2.73\left(3 \mathrm{H}, \mathrm{s}, 5-\mathrm{CH}_{3}\right)$; $4.14\left(2 \mathrm{H}, \mathrm{q}, J=7.2, \mathrm{CH}_{2} \mathrm{CH}_{3}\right) ; 7.45-7.55(6 \mathrm{H}, \mathrm{m}, \mathrm{H} \mathrm{Ph})$; 7.62-7.65 (2H, m, H Ph); 8.23-8.26 (2H, m, H Ph). ${ }^{13} \mathrm{C}$ NMR spectrum $\left(\mathrm{CDCl}_{3}\right), \delta$, ppm: $13.6 ; 23.0 ; 61.6$; 126.4 (2C); 127.9; 128.4; 128.9; 129.1; 129.3; 130.1; $132.1 ; 133.8 ; 140.6 ; 152.1 ; 159.4 ; 163.2 ; 168.5$. Found, \%: C 73.95; H 4.87; N 7.38. $\mathrm{C}_{22} \mathrm{H}_{18} \mathrm{~N}_{2} \mathrm{O}_{3}$. Calculated, \%: C 73.73; H 5.06; N 7.82.

Ethyl 5-methyl-7-(4-methoxyphenyl)-2-phenyloxazolo-

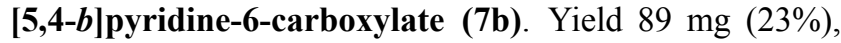
beige crystals, mp $155-156^{\circ} \mathrm{C}(i-\mathrm{PrOH})$. IR spectrum, $v, \mathrm{~cm}^{-1}: 3068,3050,3013,2985,2956,2933,2903,2837$, $1720,1622,1612,1603,1546,1518,1484,1464,1451$, $1443,1381,1371,1362,1353,1320,1294,1274,1260$, $1252,1203,1184,1161,1082,1051,1031,1021,914,835$, 782, 714, 708, 692. ${ }^{1} \mathrm{H}$ NMR spectrum $\left(\mathrm{CDCl}_{3}\right), \delta$, ppm $(J, \mathrm{~Hz}): 1.10\left(3 \mathrm{H}, \mathrm{t}, J=7.1, \mathrm{CH}_{2} \mathrm{CH}_{3}\right) ; 2.71\left(3 \mathrm{H}, \mathrm{s}, 5-\mathrm{CH}_{3}\right)$; $3.87\left(3 \mathrm{H}, \mathrm{s}, \mathrm{OCH}_{3}\right) ; 4.20\left(2 \mathrm{H}, \mathrm{q}, J=7.1, \mathrm{CH}_{2} \mathrm{CH}_{3}\right) ; 7.01-$ 7.05 (2H, m, H-3,5 Ar); 7.47-7.56 (3H, m, H Ph); 7.61$7.64(2 \mathrm{H}, \mathrm{m}, \mathrm{H}-2,6 \mathrm{Ar})$; 8.23-8.26 (2H, m, H Ph). ${ }^{13} \mathrm{C}$ NMR spectrum $\left(\mathrm{CDCl}_{3}\right), \delta$, ppm: $13.8 ; 22.9 ; 55.3$; $61.7 ; 114.0 ; 126.0 ; 126.2 ; 126.5 ; 127.9 ; 128.9 ; 130.0$; $130.9 ; 132.1 ; 140.2 ; 152.0 ; 159.3 ; 160.5 ; 162.9 ; 168.8$. Found, \%: C 70.98; H 5.23; N 7.25. $\mathrm{C}_{23} \mathrm{H}_{20} \mathrm{~N}_{2} \mathrm{O}_{4}$. Calculated, \%: C 71.12; H 5.19; N 7.21.

Ethyl 7-(4-chlorophenyl)-5-methyl-2-phenyloxazolo[5,4-b]pyridine-6-carboxylate (7c). Yield $185 \mathrm{mg}$ (47\%), light-beige crystals, $\mathrm{mp} 148-149^{\circ} \mathrm{C}(i$-PrOH). IR spectrum, $v, \mathrm{~cm}^{-1}: 3070,3034,2986,2937,2901,2871,1721,1621$, $1603,1543,1498,1484,1474,1452,1423,1382,1372$, $1362,1353,1320,1276,1257,1202,1183,1157,1091$, $1081,1073,1053,1021,1010,956,939,328,915,872$, $831,783,734,712,692 .{ }^{1} \mathrm{H}$ NMR spectrum $\left(\mathrm{CDCl}_{3}\right)$, $\delta$, ppm $(J, \mathrm{~Hz}): 1.09\left(3 \mathrm{H}, \mathrm{t}, J=7.1, \mathrm{CH}_{2} \mathrm{CH}_{3}\right) ; 2.72(3 \mathrm{H}, \mathrm{s}$, 5- $\left.\mathrm{CH}_{3}\right) ; 4.18\left(2 \mathrm{H}, \mathrm{q}, J=7.1, \mathrm{CH}_{2} \mathrm{CH}_{3}\right) ; 7.47-7.60(7 \mathrm{H}, \mathrm{m}$, $\mathrm{H} \mathrm{Ph}, \mathrm{H} \mathrm{Ar}) ; 8.22-8.24$ (2H, m, H Ph). ${ }^{13} \mathrm{C}$ NMR spectrum 
$\left(\mathrm{CDCl}_{3}\right), \delta$, ppm: $13.7 ; 23.0 ; 61.8 ; 126.2 ; 126.3 ; 127.9$; $128.8 ; 129.0 ; 130.0 ; 130.7 ; 132.2 ; 132.3 ; 135.5 ; 139.1$; $152.3 ; 159.4 ; 163.4 ; 168.3$. Found, \%: C 67.06; H 4.18; $\mathrm{N}$ 7.15. $\mathrm{C}_{22} \mathrm{H}_{17} \mathrm{ClN}_{2} \mathrm{O}_{3}$. Calculated, \%: C 67.26; $\mathrm{H}$ 4.36; N 7.13.

Ethyl 7-(4-fluorophenyl)-5-methyl-2-phenyloxazolo[5,4-b]pyridine-6-carboxylate (7d). Yield $173 \mathrm{mg}(46 \%)$, beige crystals, mp $143-144^{\circ} \mathrm{C}(i-\mathrm{PrOH})$. IR spectrum, $v, \mathrm{~cm}^{-1}: 3074,2984,2939,2903,1720,1620,1608,1544$, $1517,1483,1452,1361,1317,1273,1256,1239,1198$, $1179,1163,1079,1069,1053,1023,950,916,877,836$, $803,781,735,709,691,574 .{ }^{1} \mathrm{H}$ NMR spectrum $\left(\mathrm{CDCl}_{3}\right)$, $\delta, \operatorname{ppm}(J, \mathrm{~Hz}): 1.08\left(3 \mathrm{H}, \mathrm{t}, J=7.1, \mathrm{CH}_{2} \mathrm{CH}_{3}\right) ; 2.72(3 \mathrm{H}, \mathrm{s}$, $\left.5-\mathrm{CH}_{3}\right) ; 4.17\left(2 \mathrm{H}, \mathrm{q}, J=7.2, \mathrm{CH}_{2} \mathrm{CH}_{3}\right) ; 7.17-7.23(2 \mathrm{H}, \mathrm{m}$, $\mathrm{H}-3,5 \mathrm{Ar}) ; 7.47-7.56(3 \mathrm{H}, \mathrm{m}, \mathrm{H} \mathrm{Ph}) ; 7.62-7.65(2 \mathrm{H}, \mathrm{m}$, $\mathrm{H}-2,6 \mathrm{Ar}) ; 8.22-8.24(2 \mathrm{H}, \mathrm{m}, \mathrm{H} \mathrm{Ph}) .{ }^{13} \mathrm{C}$ NMR spectrum $\left(\mathrm{CDCl}_{3}\right), \delta, \operatorname{ppm}(J, \mathrm{~Hz}): 13.7 ; 23.0 ; 61.7 ; 115.6(\mathrm{~d}$, $J=22.5) ; 126.3(2 \mathrm{C}) ; 127.9 ; 128.9 ; 129.8$ (d, $J=8.7)$; $130.0 ; 131.4(\mathrm{~d}, J=8.7) ; 132.2 ; 139.3 ; 152.2 ; 159.4 ; 163.3$ (d, $J=249.7) ; 163.3 ; 168.4$. Found, \%: C 70.56; H 4.39; $\mathrm{N} 7.38 . \mathrm{C}_{22} \mathrm{H}_{17} \mathrm{FN}_{2} \mathrm{O}_{3}$. Calculated, \%: C 70.20; H 4.55; N 7.44.

Ethyl 5-methyl-7-(4-nitrophenyl)-2-phenyloxazolo[5,4-b]pyridine-6-carboxylate (7e). Yield $161 \mathrm{mg}(40 \%)$, colorless crystals, $\mathrm{mp} 192-193^{\circ} \mathrm{C}(i$-PrOH $)$. IR spectrum, $v, \mathrm{~cm}^{-1}: 3110,3074,3035,2988,2969,2944,2904,2856$, $1721,1622,1595,1581,1543,1516,1483,1452,1351$, $1320,1275,1256,1205,1182,1157,1108,1081,1053$, $1021,1009,945,928,916,865,859,844,782,753,723$, $711,692 .{ }^{1} \mathrm{H}$ NMR spectrum $\left(\mathrm{CDCl}_{3}\right), \delta, \mathrm{ppm}(J, \mathrm{~Hz}): 1.08$ $\left(3 \mathrm{H}, \mathrm{t}, J=7.2, \mathrm{CH}_{2} \mathrm{CH}_{3}\right) ; 2.75\left(3 \mathrm{H}, \mathrm{s}, 5-\mathrm{CH}_{3}\right) ; 4.18(2 \mathrm{H}, \mathrm{q}$, $\left.J=7.2, \mathrm{CH}_{2} \mathrm{CH}_{3}\right) ; 7.49-7.53(2 \mathrm{H}, \mathrm{m}, \mathrm{H} \mathrm{Ph}) ; 7.55-7.59$ $(1 \mathrm{H}, \mathrm{m}, \mathrm{H} \mathrm{Ph}) ; 7.79-7.82$ (2H, m, H-2,6 Ar); 8.22-8.24 $(2 \mathrm{H}, \mathrm{m}, \mathrm{H} \mathrm{Ph}) ; 8.35-8.39$ (2H, m, H-3,5 Ar). ${ }^{13} \mathrm{C}$ NMR spectrum $\left(\mathrm{CDCl}_{3}\right), \delta$, ppm: $13.8 ; 23.1 ; 62.0 ; 123.6 ; 125.9$; $126.0 ; 128.0 ; 129.0 ; 130.0 ; 130.5 ; 132.6 ; 137.7 ; 140.4$; $148.1 ; 152.7 ; 159.5 ; 164.0 ; 167.8$. Found, \%: C 65.30; $\mathrm{H}$ 4.16; N 10.53. $\mathrm{C}_{22} \mathrm{H}_{17} \mathrm{~N}_{3} \mathrm{O}_{5}$. Calculated, \%: C 65.50; $\mathrm{H} 4.25$; N 10.42 .

Synthesis of ethyl 4-aryl-2-methyl-6-oxo-5-[(phenylcarbonyl)amino]-1,6-dihydropyridine-3-carboxylates 9a-e (General method). A solution of $\mathrm{NaOH}(120 \mathrm{mg}, 3 \mathrm{mmol})$ in $\mathrm{H}_{2} \mathrm{O}(0.8 \mathrm{ml})$ was added to a suspension of oxazolo[5,4- $b$ pyridine $7 \mathbf{a}-\mathbf{e}(1 \mathrm{mmol})$ in $\mathrm{EtOH}(7 \mathrm{ml})$. The mixture was heated under reflux for $1.5 \mathrm{~h}$, then concentrated in vacuo to $1 / 3$ of its original volume. The residue was poured into $\mathrm{H}_{2} \mathrm{O}(15 \mathrm{ml})$ and acidified with $10 \%$ aqueous $\mathrm{HCl}$ to $\mathrm{pH} \sim 3$. The precipitate that formed was filtered, washed with $\mathrm{H}_{2} \mathrm{O}$, and recrystallized.

Ethyl 2-methyl-6-oxo-4-phenyl-5-[(phenylcarbonyl)amino]-1,6-dihydropyridine-3-carboxylate (9a). Yield $316 \mathrm{mg}(84 \%)$, colorless crystals, $\mathrm{mp}>250^{\circ} \mathrm{C}(i-\mathrm{PrOH})$. IR spectrum, $v \mathrm{~cm}^{-1}: 3287,3121,3051,2984,1721,1674$, $1642,1615,1517,1488,1398,1287,1201,1157,1089$, $1027,913,898,766,705,633 .{ }^{1} \mathrm{H}$ NMR spectrum (DMSO- $d_{6}$ ), $\delta$, ppm $(J, \mathrm{~Hz}): 0.76\left(3 \mathrm{H}, \mathrm{t}, J=7.1, \mathrm{CH}_{2} \mathrm{CH}_{3}\right) ; 2.33(3 \mathrm{H}, \mathrm{s}$, $\left.2-\mathrm{CH}_{3}\right) ; 3.79\left(2 \mathrm{H}, \mathrm{q}, J=7.1, \mathrm{CH}_{2} \mathrm{CH}_{3}\right) ; 7.18-7.21(2 \mathrm{H}, \mathrm{m}$, $\mathrm{H} \mathrm{Ph}) ; 7.23-7.31$ (3H, m, H Ph); 7.35-7.39 (2H, m, H Ph); 7.44-7.48 (1H, m, H Ph); 7.64-7.67 (2H, m, H Ph); 9.08 $(1 \mathrm{H}$, br. s, $5-\mathrm{NHCOPh}) ; 11.95(1 \mathrm{H}$, br. s, $1-\mathrm{NH}) .{ }^{13} \mathrm{C} \mathrm{NMR}$ spectrum $\left(\mathrm{DMSO}-d_{6}\right), \delta, \mathrm{ppm}: 12.9 ; 16.9 ; 60.2 ; 111.4$; $123.0 ; 127.1 ; 127.3$ (2C); 127.4; 127.8; 130.9; 134.1; $136.2 ; 144.4 ; 148.7 ; 159.7 ; 165.9 ; 166.0$. Found, \%: C 70.60; H 5.34; N 7.26. $\mathrm{C}_{22} \mathrm{H}_{20} \mathrm{~N}_{2} \mathrm{O}_{4}$. Calculated, \%: C 70.20; H 5.36; N 7.44.

Ethyl 4-(4-methoxyphenyl)-2-methyl-6-oxo-5-[(phenylcarbonyl)amino]-1,6-dihydropyridine-3-carboxylate (9b). Yield $300 \mathrm{mg}(74 \%)$, beige crystals, $\mathrm{mp}>250^{\circ} \mathrm{C}(\mathrm{PhMe})$. IR spectrum, $v, \mathrm{~cm}^{-1}: 3249,3132,3054,2981,2932,2907$, $2837,2781,1720,1671,1644,1611,1580,1553,1514$, 1486, 1466, 1444, 1396, 1366, 1291, 1249, 1199, 1178, 1157, 1110, 1088, 1028, 906, 872, 834, 781, 711, 692, 661, $634,583 .{ }^{1} \mathrm{H}$ NMR spectrum (DMSO- $\left.d_{6}\right), \delta, \mathrm{ppm}(J, \mathrm{~Hz})$ : $0.77\left(3 \mathrm{H}, \mathrm{t}, J=7.2, \mathrm{CH}_{2} \mathrm{CH}_{3}\right) ; 2.30\left(3 \mathrm{H}, \mathrm{s}, 2-\mathrm{CH}_{3}\right) ; 3.68$ $\left(3 \mathrm{H}, \mathrm{s}, \mathrm{OCH}_{3}\right) ; 3.82\left(2 \mathrm{H}, \mathrm{q}, J=7.2, \mathrm{CH}_{2} \mathrm{CH}_{3}\right) ; 6.87(2 \mathrm{H}, \mathrm{d}$, $J=8.8, \mathrm{H}-3,5 \mathrm{Ar}) ; 7.12(2 \mathrm{H}, \mathrm{d}, J=8.8, \mathrm{H}-2,6 \mathrm{Ar}) ; 7.38$ $7.44(2 \mathrm{H}, \mathrm{m}, \mathrm{H} \mathrm{Ph}) ; 7.46-7.52(1 \mathrm{H}, \mathrm{m}, \mathrm{H} \mathrm{Ph}) ; 7.73(2 \mathrm{H}, \mathrm{d}$, $J=7.4$, H Ph); 9.32 (1H, br. s, 5-NHCOPh); 12.21 (1H, br. s, $1-\mathrm{NH}) .{ }^{13} \mathrm{C}$ NMR spectrum (DMSO- $\left.d_{6}\right), \delta$, ppm: $13.3 ; 17.2$; $55.1 ; 60.5 ; 111.7 ; 113.2 ; 123.2 ; 127.4 ; 128.2 ; 128.5 ; 128.7$; $131.3 ; 134.1 ; 144.4 ; 148.6 ; 158.9 ; 160.1 ; 166.1 ; 166.3$. Found, \%: C 67.78; H 5.33; N 6.72. $\mathrm{C}_{23} \mathrm{H}_{22} \mathrm{~N}_{2} \mathrm{O}_{5}$. Calculated, \%: C 67.97; H 5.46; N 6.89.

Ethyl 4-(4-chlorophenyl)-2-methyl-6-oxo-5-[(phenylcarbonyl)amino]-1,6-dihydropyridine-3-carboxylate (9c). Yield $366 \mathrm{mg}(89 \%)$, light-beige crystals, $\mathrm{mp}>250^{\circ} \mathrm{C}$ (i-PrOH). IR spectrum, $v, \mathrm{~cm}^{-1}: 3288,3127,3056,2981$, $2928,2869,2788,1722,1674,1642,1606,1582,1571$, $1556,1517,1487,1445,1393,1366,1286,1247,1200$, $1188,1157,1087,1027,1015,904,871,832,822,787$, $707,691,662,632 .{ }^{1} \mathrm{H}$ NMR spectrum (DMSO- $\left.d_{6}\right), \delta$, ppm $(J, \mathrm{~Hz}): 0.75\left(3 \mathrm{H}, \mathrm{t}, J=7.1, \mathrm{CH}_{2} \mathrm{CH}_{3}\right) ; 2.34\left(3 \mathrm{H}, \mathrm{s}, 2-\mathrm{CH}_{3}\right)$; $3.81\left(2 \mathrm{H}, \mathrm{q}, J=7.1, \mathrm{CH}_{2} \mathrm{CH}_{3}\right) ; 7.18(2 \mathrm{H}, \mathrm{d}, J=8.4, \mathrm{H}-2,6$ Ar); 7.38-7.43 (4H, m, H Ph, H-3,5 Ar); $7.50(1 \mathrm{H}, \mathrm{t}$, $J=7.3, \mathrm{H} \mathrm{Ph}) ; 7.70(2 \mathrm{H}, \mathrm{d}, J=7.4, \mathrm{H} \mathrm{Ph}) ; 9.37(1 \mathrm{H}$, br. s, 5-NHCOPh); 12.34 (1H, br. s, 1-NH). ${ }^{13} \mathrm{C}$ NMR spectrum (DMSO- $d_{6}$ ), $\delta$, ppm: $13.2 ; 17.4 ; 60.5 ; 110.7 ; 123.4 ; 127.4$; $127.8 ; 128.2 ; 129.2 ; 131.4 ; 132.5 ; 133.9 ; 135.3 ; 145.5$; $147.7 ; 159.9 ; 165.9 ; 166.0$. Found, \%: C 64.13; H 4.75; $\mathrm{N}$ 6.90. $\mathrm{C}_{22} \mathrm{H}_{19} \mathrm{ClN}_{2} \mathrm{O}_{4}$. Calculated, \%: C 64.32; H 4.66; N 6.82 .

Ethyl 4-(4-fluorophenyl)-2-methyl-6-oxo-5-[(phenylcarbonyl)aminol-1,6-dihydropyridine-3-carboxylate (9d). Yield $315 \mathrm{mg}(80 \%)$, beige crystals, $\mathrm{mp}>250^{\circ} \mathrm{C}(i$-PrOH $)$. IR spectrum, $v, \mathrm{~cm}^{-1}: 3280,3127,3057,2982,2932,2870$, $2789,1721,1674,1643,1611,1595,1582,1556,1512$, $1486,1446,1410,1396,1367,1288,1229,1200,1155$, 1115, 1088, 1027, 1015, 903, 837, 800, 787, 710, 663, 635. ${ }^{1} \mathrm{H}$ NMR spectrum (DMSO-d $\left.d_{6}\right), \delta$, ppm $(J, \mathrm{~Hz}): 0.76(3 \mathrm{H}, \mathrm{t}$, $\left.J=7.0, \mathrm{CH}_{2} \mathrm{CH}_{3}\right) ; 2.33\left(3 \mathrm{H}, \mathrm{s}, 2-\mathrm{CH}_{3}\right) ; 3.81(2 \mathrm{H}, \mathrm{q}, J=7.0$, $\left.\mathrm{CH}_{2} \mathrm{CH}_{3}\right) ; 7.14-7.22(4 \mathrm{H}, \mathrm{m}, \mathrm{H} \mathrm{Ar}) ; 7.35-7.45(2 \mathrm{H}, \mathrm{m}$, $\mathrm{H} \mathrm{Ph}) ; 7.46-7.54(1 \mathrm{H}, \mathrm{m}, \mathrm{H} \mathrm{Ph}) ; 7.69(2 \mathrm{H}, \mathrm{d}, J=7.4$, $\mathrm{H} \mathrm{Ph}) ; 9.36$ (1H, br. s, 5-NHCOPh); 12.31 (1H, br. s, 1-NH). ${ }^{13} \mathrm{C}$ NMR spectrum (DMSO- $\left.d_{6}\right), \delta, \operatorname{ppm}(J, \mathrm{~Hz}): 13.3 ; 17.3$; $60.5 ; 111.1 ; 114.6(\mathrm{~d}, J=21.7) ; 123.4 ; 127.4 ; 128.2 ; 129.5$ $(\mathrm{d}, J=7.8) ; 131.4 ; 132.8(\mathrm{~d}, J=3.5) ; 134.0 ; 145.2 ; 148.0$; $160.0 ; 161.7$ (d, $J=244.5)$; 166.0 (2C). Found, \%: C 67.10; $\mathrm{H}$ 4.76; N 7.04. $\mathrm{C}_{22} \mathrm{H}_{19} \mathrm{FN}_{2} \mathrm{O}_{4}$. Calculated, \%: C 67.00; H 4.86; N 7.10. 
Ethyl 2-methyl-4-(4-nitrophenyl)-6-oxo-5-[(phenylcarbonyl)amino]-1,6-dihydropyridine-3-carboxylate (9e). Yield $396 \mathrm{mg}$ (94\%), colorless crystals, mp $229-230^{\circ} \mathrm{C}$ (i-PrOH). IR spectrum, $v, \mathrm{~cm}^{-1}: 3280,3119,2982,2940$, 2893, 2842, 2763, 2661, 1721, 1665, 1634, 1595, 1515, $1476,1446,1385,1346,1292,1285,1263,1214,1171$, 1114, 1089, 1032, 965, 841, 782, 735, 689. ${ }^{1} \mathrm{H}$ NMR spectrum (DMSO- $\left.d_{6}\right), \delta$, ppm $(J, \mathrm{~Hz}): 0.68(3 \mathrm{H}, \mathrm{t}, J=7.1$, $\left.\mathrm{CH}_{2} \mathrm{CH}_{3}\right) ; 2.27\left(3 \mathrm{H}, \mathrm{s}, 2-\mathrm{CH}_{3}\right) ; 3.75(2 \mathrm{H}, \mathrm{q}, J=7.1$, $\left.\mathrm{CH}_{2} \mathrm{CH}_{3}\right) ; 7.20-7.29(4 \mathrm{H}, \mathrm{m}, \mathrm{H} \mathrm{Ph}, \mathrm{H}-2,6 \mathrm{Ar}) ; 7.33(2 \mathrm{H}, \mathrm{t}$, $J=7.4, \mathrm{H} \mathrm{Ph}) ; 7.38-7.45(1 \mathrm{H}, \mathrm{m}, \mathrm{H} \mathrm{Ph}) ; 8.20(2 \mathrm{H}, \mathrm{d}$, $J=8.6, \mathrm{H}-3,5 \mathrm{Ar}) ; 9.44$ (1H, br. s, 5-NHCOPh); $12.00(1 \mathrm{H}$, br. s, $1-\mathrm{NH}) .{ }^{13} \mathrm{C}$ NMR spectrum (DMSO- $\left.d_{6}\right), \delta$, ppm: 13.2; $13.8 ; 60.3 ; 109.7 ; 122.9 ; 126.9 ; 128.2 ; 129.5 ; 130.4 ; 132.2$; $133.5 ; 134.0 ; 141.1 ; 144.8 ; 146.4 ; 157.0 ; 160.2 ; 165.8$. Found, \%: C 62.51; H 4.58; N 10.05. $\mathrm{C}_{22} \mathrm{H}_{19} \mathrm{~N}_{3} \mathrm{O}_{6}$. Calculated, \%: C 62.70; H 4.54; N 9.97.

$\mathrm{X}$-ray structural analysis of compound 4a. Crystals suitable for X-ray structural analysis were obtained by slow evaporation of a solution of compound $4 \mathbf{a}$ in benzene at room temperature. The set of reflections was obtained on an Xcalibur Ruby (Agilent technologies, UK) diffractometer with a CCD detector according to the standard routine $(\mathrm{MoK} \alpha$ radiation, $295(2) \mathrm{K}, \omega$-scanning with a step of $1^{\circ}$ ). Absorption was corrected empirically using the SCALE3 ABSPACK algorithm. ${ }^{38}$ The structure was solved using the SHELXS ${ }^{39}$ program and refined using the SHELXL ${ }^{40}$ program with the OLEX2 graphical interface. ${ }^{41}$ The positions of the $\mathrm{H}$ atoms was refined using the rider model. The positions of the $\mathrm{H}$ atoms of the $\mathrm{NH}$ groups were refined independently in the isotropic approximation. The crystals of compound $4 \mathrm{a}\left(\mathrm{C}_{22} \mathrm{H}_{22} \mathrm{~N}_{2} \mathrm{O}_{4}, M 378.41\right)$ are monoclinic, spatial symmetry group $P 2{ }_{1} / \mathrm{c} ; a 10.433(3)$, b 9.748(2), c 20.107(6) $\AA$; $\beta$ 99.60(3) ${ }^{\circ} ; V$ 2016.1(10) $\AA^{3}$; $Z 4 ; d_{\text {calc }} 1.247 \mathrm{~g} / \mathrm{cm}^{3} ; \mu 0.086 \mathrm{~mm}^{-1}$. The final refinement parameters: $R_{1} 0.0660$ (for 2716 reflections with $I>2 \sigma(I)$ ), $w R_{2} \quad 0.2089$ (for all 4801 independent reflections, $\left.R_{\text {int }} 0.0417\right), S 1.021$. The X-ray structural analysis data was deposited at the Cambridge Crystallographic Data Center (deposit CCDC 2096420).

Supplementary information file containing ${ }^{1} \mathrm{H}$ and ${ }^{13} \mathrm{C}$ NMR spectra of all new compounds is available at the journal website at http://link.springer.com/journal/10593.

The study was carried out with the financial support of the Russian Foundation for Basic Research within the framework of scientific project No. 19-33-90229.

\section{References}

1. (a) Sośnicki, J. G.; Idzik, T. J. Synthesis 2019, 3369. (b) Przhevalskii, N. M.; Laypanov, R. K.; Tokmakov, G. P.; Lukina, I. V.; Vershinkin, D. A.; Tafeenko, V. A. Russ. Chem. Bull., Int. Ed. 2021, 70, 555. [Izv. Akad. Nauk, Ser. Khim. 2021, 555.]

2. Fearon, D.; Westwood, I. M.; van Montfort, R. L. M.; Bayliss, R.; Jones, K.; Bavetsias, V. Bioorg. Med. Chem. 2018, 26, 3021.

3. Lim, N.-K.; Zhang, H.; Sowell, C. G.; Gosselin, F. Tetrahedron Lett. 2020, 61, 152447.
4. Crawford, J. J.; Lee, W.; Johnson, A. R.; Delatorre, K. J.; Chen, J.; Eigenbrot, C.; Heidmann, J.; Kakiuchi-Kiyota, S.; Katewa, A.; Kiefer, J. R.; Liu, L.; Lubach, J. W.; Misner, D.; Purkey, H.; Reif, K.; Vogt, J.; Wong, H.; Yu, C.; Young, W. B. ACS Med. Chem. Lett. 2020, 11, 1588.

5. Semple, G.; Ashworth, D. M.; Baker, G. R.; Batt, A. R.; Baxter, A. J.; Benzies, D. W.; Elliot, L. H.; Evans, D. M.; Franklin, R. J.; Hudson, P.; Jenkins, P. D.; Pitt, G. R.; Rooker, D. P.; Sheppard, A.; Szelke, M.; Yamamoto, S.; Isomura, Y. Bioorg. Med. Chem. Lett. 1997, 7, 1337.

6. Bernstein, P. R.; Andisik, D.; Bradley, P. K.; Bryant, C. B.; Ceccarelli, C.; Damewood, J. R., Jr.; Earley, R.; Edwards, P. D.; Feeney, S.; Gomes, B. C.; Kosmider, B. J.; Steelman, G. B.; Thomas, R. M.; Vacek, E. P.; Veale, C. A.; Williams, J. C.; Wolanin, D. J.; Woolson, S. A. J. Med. Chem. 1994, 37, 3313.

7. Zhang, L.; Lin, D.; Sun, X.; Curth, U.; Drosten, C.; Sauerhering, L.; Becker, S.; Rox, K.; Hilgenfeld, R. Science 2020, 368, 409.

8. Mengist, H. M.; Fan, X.; Jin, T. Signal Transduction Targeted Ther. 2020, 5, 67.

9. Kusakabe, K.-i.; Tada, Y.; Iso, Y.; Sakagami, M.; Morioka, Y.; Chomei, N.; Shinonome, S.; Kawamoto, K.; Takenaka, H.; Yasui, K.; Hamana, H.; Hanasaki, K. Bioorg. Med. Chem. 2013, 21, 2045.

10. Gado, F.; Mannelli, L. D. C.; Lucarini, E.; Bertini, S.; Cappelli, E.; Digiacomo, M.; Stevenson, L. A.; Macchia, M.; Tuccinardi, T.; Ghelardini, C.; Pertwee, R. G.; Manera C. J. Med. Chem. 2019, 62, 276.

11. Li, Y. H.; Tseng, P.-S.; Evans, K. A.; Jaworski, J.-P.; Morrow, D. M.; Fries, H. E.; Wu, C. W.; Edwards, R. M.; Jin, J. Bioorg. Med. Chem. Lett. 2010, $20,6744$.

12. Meesters, C.; Mönig, T.; Oeljeklaus, J.; Krahn, D.; Westfall, C. S.; Hause, B.; Jez, J. M.; Kaiser, M.; Kombrink, E. Nat. Chem. Biol. 2014, 10, 830.

13. Ward, A.; Brogden, R. N.; Heel, R. C.; Speight, T. M.; Avery, G. S. Drugs 1983, 26, 468.

14. Kulakov, I. V.; Shatsauskas, A. L.; Matsukevich, M. V.; Palamarchuk, I. V.; Seilkhanov, T. M.; Gatilov, Yu. V.; Fisyuk, A. S. Synthesis 2017, 3700.

15. Kulakov, I. V.; Matsukevich, M. V.; Levin, M. L.; Palamarchuk, I. V.; Seilkhanov, T. M.; Fisyuk, A. S. Synlett 2018, 1741.

16. Palamarchuk, I. V.; Matsukevich, M. V.; Kulakov, I. V.; Seilkhanov, T. M.; Fisyuk, A. S. Chem. Heterocycl. Compd. 2019, 55, 788. [Khim. Geterotsikl. Soedin. 2019, 55, 788.]

17. Kulakov, I. V.; Matsukevich, M. V.; Shulgau, Z. T.; Sergazy, S.; Seilkhanov, T. M.; Puzari, A.; Fisyuk, A. S. Chem. Heterocycl. Compd. 2015, 51, 991. [Khim. Geterotsikl. Soedin. 2015, 51, 991.]

18. Kulakov, I. V.; Palamarchuk, I. V.; Shulgau, Z. T.; Seilkhanov, T. M.; Gatilov, Yu. V.; Fisyuk, A. S. J. Mol. Struct. 2018, 1166, 262.

19. Shatsauskas, A. L.; Abramov, A. A.; Chernenko, S. A.; Kostyuchenko, A. S.; Fisyuk, A. S. Synthesis 2020, 227.

20. Shatsauskas, A.; Shatalin, Yu.; Shubina, V.; Zablodtskii, Y.; Chernenko, S.; Samsonenko, A.; Kostyuchenko, A.; Fisyuk, A. Dyes Pigm. 2021, 187, 109072.

21. Fisyuk, A. S.; Kostyuchenko, A. S.; Goncharov, D. S. Russ. J. Org. Chem. 2020, 56, 1863. [Zh. Org. Khim. 2020, 56, 1649.]

22. Shatsauskas, A. L.; Abramov, A. A.; Saibulina, E. R.; Palamarchuk, I. V.; Kulakov, I. V.; Fisyuk, A. S. Chem. Heterocycl. Compd. 2017, 53, 186. [Khim. Geterotsikl. Soedin. 2017, 53, 186.]

23. Vanden Eynde, J. J.; Labuche, N.; Van Haverbeke, Y. Synth. Commun. 1997, 27, 3683. 
24. Maquestiau, A.; Vanden Eynde, J.-J.; Papleux, P. Bull. Soc. Chim. Belg. 1985, 94, 849.

25. Cunha, S.; Filho, R. F. dos S.; Saraiva, K. H.; AzevedoSantos, A. V.; Menezes, D. Tetrahedron Lett. 2013, 54, 3366.

26. Wang, S.; Sun, J.; Yu, G.; Hu, X.; Liu, J. O.; Hu, Y. Org. Biomol. Chem. 2004, 2, 1573.

27. Yonezawa, Y.; Konn, A.; Shin, C.-g. Heterocycles 2004, 63, 2735.

28. Hussenether, T.; Troschütz, R. J. Heterocycl. Chem. 2004, 41, 857.

29. Rao, Y. S. J. Org. Chem. 1976, 41, 722.

30. Wang, S.; Pan, J.; Hu, Y.; Hu, H. Synthesis 2005, 753.

31. Worayuthakarn, R.; Nealmongkol, P.; Ruchirawat, S.; Thasana, N. Tetrahedron 2012, 68, 2864.

32. Liu, J.; Cremosnik, G. S.; Otte, F.; Pahl, A.; Sievers, S.; Strohmann, C.; Waldmann, H. Angew. Chem., Int. Ed. 2021, $60,4648$.

33. Hamama, W. S.; Ibrahim, M. E.; Zoorob, H. H. Arch. Pharm. Chem. Life Sci. 2012, 345, 468.
34. Shatsauskas, A. L.; Mamonova, T. E.; Stasyuk, A. J.; Chernenko, S. A.; Slepukhin, P. A.; Kostyuchenko, A. S.; Fisyuk, A. S. J. Org. Chem. 2020, 85, 10072.

35. (a) Kumar, A.; Singh, S.; Mudahar, G. S.; Thind, K. S. Radiat. Phys. Chem. 2006, 75, 737. (b) Williams, A. T. R.; Winfield, S. A.; Miller, J. N. Analyst 1983, 108, 1067.

36. (a) Allen, M. W. Measurement of Fluorescence Quantum Yields; Thermo Fisher Scientific: Madison. Technical note: 52019. (b) Brouwer, A. M. Pure Appl. Chem. 2011, 83, 2213.

37. (a) R., C.; Pise, A.; Shah, S. K.; D., R.; V., S.; Tiwari, K. N. Org. Lett. 2020, 22, 6557. (b) Xia, B.; Chen, W.; Zhao, Q.; Yu, W.; Chang, J. Org. Lett. 2019, 21, 2583.

38. CrysAlisPro, Version 1.171.37.33 (release 27-03-2014); Agilent Technologies.

39. Sheldrick, G. M. Acta Crystallogr., Sect. A: Found. Crystallogr. 2008, A64, 112.

40. Sheldrick, G. M. Acta Crystallogr., Sect. C: Struct. Chem. 2015, $C 71,3$.

41. Dolomanov, O. V.; Bourhis, L. J.; Gildea, R. J.; Howard, J. A. K.; Puschmann, H. J. Appl. Crystallogr. 2009, 42, 339. 